\title{
QUADRATIC SPLINE SUBDOMAIN METHOD FOR VOLTERRA INTEGRAL EQUATIONS
}

\author{
V. DEPUTAT, P. OJA and D. SAVELJEVA \\ University of Tartu, Institute of Applied Mathematics, Estonia \\ Liivi 2, Tartu, 50409, Estonia \\ E-mail: viktoria.deputat@ut.ee; peeter.oja@ut.ee \\ E-mail: darja.saveljeva@ut.ee
}

Received June 6, 2005; revised November 28, 2005

\begin{abstract}
In our work we consider the step-by-step and nonlocal subdomain methods with quadratic splines. We prove that the first method is unstable. In the case of nonlocal method we replaced the first derivative condition by a not-a-knot boundary condition at the other end of the interval of integration. As a result, we get stability of this method. Main results about stability and convergence are based on the uniform boundedness of quadratic spline histopolation projections. The numerical tests given at the end support the theoretical results.

Key words: quadratic spline histopolation, spline projections, Volterra integral equations, stability and convergence of spline subdomain methods
\end{abstract}

\section{Introduction}

One of the most practical methods for solving Volterra integral equations of the second kind is the polynomial spline collocation with step-by-step implementation. This method is known to be unstable for cubic and higher order smooth splines (see $[4,6,10,11])$. In the case of quadratic splines of class $C^{1}$ the stability region consists only of one point $[11]$. In $[12,13]$ one of the initial conditions, which are required by the standard quadratic and cubic spline collocation, is replaced by a not-a-knot boundary condition at the other end of the interval. These methods cannot be any more implemented step-by-step and, in the case of linear integral equation, need the solution of a linear system which can be successfully done by the Gaussian elimination. On the other hand, the nonlocal method with quadratic splines gives the stability in the whole interval of collocation parameter and in the case of cubic splines this method gives stability in the same interval of collocation parameter as in the case of the traditional collocation with linear splines [11].

It is natural to use a subdomain method when equation's free term is given by average values in subintervals. In addition, the collocation method can be considered 
as a subdomain method's approximation. Thus, the subdomain method is a basic method in collocation methods theory.

In this paper we study the use of quadratic splines in two particular cases of subdomain methods. First we show that the traditional step-by-step implementation gives an unstable method. The replacement of one of initial conditions by the nota-knot condition at the other end of the interval of integration results in converging method for quite wide class of integral equations, including, for example, those with weakly singular kernel. We consider both cases as projection methods and use a general convergence theorem for operator equations to establish the convergence of nonlocal method with two-sided estimates.

\section{Step-by-Step Method}

Consider the Volterra integral equation

$$
y(t)=\int_{0}^{t} \mathcal{K}(t, s, y(s)) d s+f(t), \quad t \in[0, T],
$$

with given functions $f:[0, T] \rightarrow \mathbb{R}, \mathcal{K}: S \times \mathbb{R} \rightarrow \mathbb{R}$ and the set $S=\{(t, s):$ $0 \leq s \leq t \leq T\}$.

There will be used a mesh $\Delta_{N}: 0=t_{0}<t_{1}<\ldots<t_{N}=T$ which represents spline knots. As we consider the process $N \rightarrow \infty$, the knots $t_{i}$ depend on $N$. In order to determine the approximate solution $u$ of the equation (2.1) as quadratic spline of class $C^{1}$ (denote this space by $S_{2}\left(\Delta_{N}\right)$ ), we impose the following subdomain conditions

$$
\int_{t_{i-1}}^{t_{i}} u(t) d t=\int_{t_{i-1}}^{t_{i}} \int_{0}^{t} \mathcal{K}(t, s, u(s)) d s d t+\int_{t_{i-1}}^{t_{i}} f(t) d t, \quad i=1, \ldots, N .
$$

To be able to start the calculations of this method, assume that we can use the initial values

$$
u(0)=y(0), \quad u^{\prime}(0)=y^{\prime}(0),
$$

which are justifi ed by the requirement $u \in C^{\mathbb{1}}[0, T]$. Thus, on every interval $\left[t_{i-1}, t_{i}\right]$ we have two conditions of smoothness and one subdomain condition to determine three parameters of $u$ as a polynomial of degree two on $\left[t_{i-1}, t_{i}\right]$. This allows us to implement the method step-by-step, progressing from the interval $\left[t_{i-1}, t_{i}\right]$ to the next one.

Let us introduce the vector subspace of $C[0, T]$ :

$$
C_{0}[0, T]=\left\{f \in C[0, T]: \exists f^{\prime}(0)=\lim _{h \rightarrow 0+} \frac{f(h)-f(0)}{h}\right\} .
$$

For any $f \in C_{0}[0, T]$, let $P_{N} f \in S_{2}\left(\Delta_{N}\right)$ be such that

$$
\left\{\begin{array}{l}
\left(P_{N} f\right)(0)=f(0), \quad\left(P_{N} f\right)^{\prime}(0)=f^{\prime}(0), \\
\int_{t_{i-1}}^{t_{i}}\left(P_{N} f\right)(t) d t=\int_{t_{i-1}}^{t_{i}} f(t) d t, \quad i=1, \ldots, N .
\end{array}\right.
$$


Let the mesh $\Delta_{N}$ be complemented with knots $t_{-2}<t_{-1}<t_{0}$ and $t_{N+2}>t_{N+1}>t_{N}$. Denote $h_{i}=t_{i}-t_{i-1}, i=-1, \ldots, N+2$. This enables us to introduce the normalized B-splines, for $i=-1, \ldots, N$,

$$
B_{i}(t)= \begin{cases}\frac{\left(t-t_{i-1}\right)^{2}}{h_{i}\left(h_{i}+h_{i+1}\right)}, & t \in\left[t_{i-1}, t_{i}\right), \\ \frac{\left(t_{i+1}-t\right)\left(t-t_{i-1}\right)}{h_{i+1}\left(h_{i}+h_{i+1}\right)}+\frac{\left(t_{i+2}-t\right)\left(t-t_{i}\right)}{h_{i+1}\left(h_{i+1}+h_{i+2}\right)}, & t \in\left[t_{i}, t_{i+1}\right), \\ \frac{\left(t_{i+2}-t\right)^{2}}{h_{i+2}\left(h_{i+1}+h_{i+2}\right)}, & t \in\left[t_{i+1}, t_{i+2}\right) .\end{cases}
$$

If we use the representation $\left(P_{N} f\right)(t)=\sum_{-1 \leq i \leq N+1} c_{i} B_{i}(t), t \in[0, T]$, the conditions (2.4) could be written equivalently as

$$
\left\{\begin{array}{l}
c_{-1}=f(0)-\frac{h_{0}}{2} f^{\prime}(0), \\
h_{1} c_{-1}+h_{0} c_{0}=\left(h_{0}+h_{1}\right) f(0), \\
c_{i-2} \int_{t_{i-1}}^{t_{i}} B_{i-2}(t) d t+c_{i-1} \int_{t_{i-1}}^{t_{i}} B_{i-1}(t) d t+c_{i} \int_{t_{i-1}}^{t_{i}} B_{i}(t) d t=\int_{t_{i-1}}^{t_{i}} f(t) d t, \\
i=1, \ldots, N .
\end{array}\right.
$$

This linear system to determine the coeffi cients $c_{j}$ has a lower tridiagonal matrix with nonzero entries on main diagonal. Thus, the projector $P_{N}$ is correctly defi ned.

Consider the integral operator defi ned by

$$
(K u)(t)=\int_{0}^{t} \mathcal{K}(t, s, u(s)) d s, \quad t \in[0, T] .
$$

Lemma 1. The spline subdomain problem (2.2), (2.3) is equivalent to the equation

$$
u=P_{N} K u+P_{N} f, u \in S_{2}\left(\Delta_{N}\right),
$$

provided the kernel $\mathcal{K}$ in $(2.5)$ is continuous and differentiable with respect to $t$ in some neighbourhood of zero.

Proof. Note that in the assumptions about $\mathcal{K}$ it holds $K: C[0, T] \rightarrow C_{0}[0, T]$. The proof of Lemma 1 is a standard calculation based on the property of $P_{N}$ that $P_{N} f=0$ if and only if

$$
f(0)=0, \quad f^{\prime}(0)=0, \quad \int_{t_{i-1}}^{t_{i}} f(t) d t=0, \quad i=1, \ldots, N .
$$

Indeed, then (2.6) is equivalent to the equalities

$$
\begin{gathered}
(u-K u-f)(0)=0, \quad(u-K u-f)^{\prime}(0)=0 \\
\int_{t_{i-1}}^{t_{i}}(u-K u-f)(t) d t=0, \quad i=1, \ldots, N
\end{gathered}
$$


The first one of them is equivalent to $u(0)=f(0)$ or $u(0)=y(0)$ because $y(0)=$ $f(0)$. The integral equalities are just (2.2). Taking into account (2.1), we get that $(u-K u-f)^{\prime}(0)=0$ is equivalent to $u^{\prime}(0)-(K u)^{\prime}(0)=y^{\prime}(0)-(K y)^{\prime}(0)$ or $u^{\prime}(0)-\mathcal{K}(0,0, u(0))=y^{\prime}(0)-\mathcal{K}(0,0, y(0))$ (in fact, we use the differentiability of $\mathcal{K}$ with respect to $t$ in some neighbourhood of 0 to ensure the existence of $(K u)^{\prime}(0)$ and $\left.(K y)^{\prime}(0)\right)$. But the last equality, as $u(0)=y(0)$, is equivalent to $u^{\prime}(0)=y^{\prime}(0)$ which completes the proof.

Suppose in the sequel throughout this section that the mesh is uniform, i.e.

$$
t_{i}-t_{i-1}=h=\frac{T}{N}, \quad i=1, \ldots, N
$$

DEFINITION 1. We say that the spline subdomain method with quadratic splines is stable if for any $\lambda \in \mathbb{C}$ and any $f \in C^{1}[0, T]$, the approximate solution $u \in S_{2}\left(\Delta_{N}\right)$ of the test equation

$$
y(t)=\lambda \int_{0}^{t} y(s) d s+f(t), \quad t \in[0, T],
$$

remains bounded in $L_{\infty}(0, T)$ as $h \rightarrow 0$.

Proposition 1. The quadratic spline subdomain method is not stable.

Proof. Suppose that the method is stable. For $\lambda=0$, the approximate solution $u$ of the test equation is just $P_{N} f$. Then the principle of uniform boundedness yields that the sequence $\left\|P_{N}\right\|_{C^{1} \rightarrow L_{\infty}}$ is bounded, i.e., for all $f \in C^{1}[0, T]$,

$$
\|u\|_{\infty}=\left\|P_{N} f\right\|_{\infty} \leq \mathrm{const}\|f\|_{C^{1}[0, T]}
$$

where the constant may depend on $T$ and $\lambda$, but not on $N$.

Assume that the mesh $\Delta_{N}$ is complemented with knots $t_{i}=i h, i=-2,-1$, and $i=N+1, N+2$. Then the B-splines considered above are

$$
B_{i}(t)=\frac{1}{2 h^{2}} \begin{cases}\left(t-t_{i-1}\right)^{2}, & t \in\left[t_{i-1}, t_{i}\right), \\ 2 h^{2}-\left(t_{i+1}-t\right)^{2}-\left(t-t_{i}\right)^{2}, & t \in\left[t_{i}, t_{i+1}\right), \\ \left(t_{i+2}-t\right)^{2}, & t \in\left[t_{i+1}, t_{i+2}\right) .\end{cases}
$$

Given any function $f \in C_{0}[0, T]$, the coeffi cients $c_{j}$ of $u=P_{N} f=\sum_{-1 \leq j \leq N} c_{j} B_{j}$ via (2.4) are determined by the system

$$
\left(\begin{array}{cccccc}
1 & 0 & 0 & 0 & \cdots & 0 \\
1 & 1 & 0 & 0 & \cdots & 0 \\
1 & 4 & 1 & 0 & \cdots & 0 \\
& \ddots & \ddots & \ddots & & \\
& & & & & \\
0 & \cdots & 0 & 1 & 4 & 1
\end{array}\right)\left(\begin{array}{c}
c_{-1} \\
c_{0} \\
c_{1} \\
\vdots \\
c_{N}
\end{array}\right)=\left(\begin{array}{c}
g_{-1} \\
g_{0} \\
g_{1} \\
\vdots \\
\\
g_{N}
\end{array}\right)
$$

where 


$$
g_{-1}=f(0)-\frac{h}{2} f^{\prime}(0), \quad g_{0}=2 f(0), \quad g_{i}=\frac{6}{h} \int_{t_{i-1}}^{t_{i}} f(t) d t, \quad i=1, \ldots, N .
$$

Consider the equations of (2.8) (except fi rst two of them) as the difference equation

$$
c_{i-2}+4 c_{i-1}+c_{i}=g_{i}, \quad i=1, \ldots, N .
$$

Its characteristic equation $\lambda^{2}+4 \lambda+1=0$ has the roots $\lambda=-2-\sqrt{3}$ and $\mu=$ $-2+\sqrt{3}$.

Take the vector $g=(\varepsilon / 2, \varepsilon, 0, \ldots, 0)^{T}$ and look for

$$
c_{i}=K_{1} \lambda^{i+1}+K_{2} \mu^{i+1}, \quad i=-1, \ldots, N,
$$

as the corresponding solution of the system (2.8). First two equations of (2.8) allow to determine the coeffi cients $K_{1}=\varepsilon(2-\mu) /(2(\lambda-\mu))$ and $K_{2}=\varepsilon(2-\lambda) /(2(\mu-\lambda))$. Also, for $i \geq 1$, we have

$$
\left|c_{i}\right|=\left|\lambda^{i}\left(K_{1} \lambda+K_{2} \mu\left(\frac{\mu}{\lambda}\right)^{i}\right)\right| \geq \text { const } \varepsilon|\lambda|^{i} .
$$

Consider the function $f(t)=h \cos (\pi t / h), t \in[0, T]$. Then

$$
f(0)=h, \quad f^{\prime}(0)=0, \quad \int_{t_{i-1}}^{t_{i}} f(t) d t=0, \quad i=1, \ldots, N .
$$

It is also easy to verify that $\|f\|_{C^{1}} \leq$ const. Letting $\varepsilon=h$, the use of (2.9) and the stability of B-splines (see [14]) gives

$$
\|u\|_{\infty} \geq \text { const }\left|c_{N}\right| \geq \operatorname{const} \varepsilon|\lambda|^{N}=\text { const } \frac{|\lambda|^{N}}{N} \rightarrow \infty,
$$

as $N \rightarrow \infty$, which contradicts (2.7). Proposition is proved.

Remark. The proof of Proposition 1 shows that the influence of round-off errors (whose role may be played, e.g., by $\varepsilon$ ) increases unboundedly when $N$ goes to infi nity.

\section{Nonlocal Method}

As we have seen in the previous section, the subdomain method with step-by-step implementation is unstable. A hopeful reparation which works in collocation could be the replacement of the fi rst derivative condition by a not-a-knot boundary condition at the other end of the interval of integration, so in this case additional conditions are

$$
\begin{aligned}
& u(0)=y(0), \\
& u^{\prime \prime}\left(t_{N-1}-0\right)=u^{\prime \prime}\left(t_{N-1}+0\right) .
\end{aligned}
$$

Let note that we are considering arbitrary mesh $\Delta_{N}$ with $h_{i}=t_{i}-t_{i-1}$. Let the operator $P_{N}: C[0, T] \rightarrow C[0, T]$ be such that, for any $f \in C[0, T]$, we have $P_{N} f \in S_{2}\left(\Delta_{N}\right)$ and 


$$
\begin{aligned}
& \left(P_{N} f\right)(0)=f(0), \\
& \int_{t_{i-1}}^{t_{i}}\left(P_{N} f\right)(t) d t=\int_{t_{i-1}}^{t_{i}} f(t) d t, i=1, \ldots, N, \\
& \left(P_{N} f\right)^{\prime \prime}\left(t_{N-1}-0\right)=\left(P_{N} f\right)^{\prime \prime}\left(t_{N-1}+0\right) .
\end{aligned}
$$

Firstly we have to verify that such a spline $P_{N} f$ exists and is uniquely determined.

Denote $z_{i}=h_{i}^{-1} \int_{t_{i-1}}^{t_{i}} f(t) d t, i=1, \ldots, N$. Look for a cubic (interpolating) spline $S \in S_{3}\left(\Delta_{N}\right)$ satisfying

$$
\begin{aligned}
& S(0)=0, \quad S^{\prime}(0)=f(0), \\
& S\left(t_{i}\right)=\sum_{j=1}^{i} h_{j} z_{j}, i=1, \ldots, N, \\
& S^{\prime \prime \prime}\left(t_{N-1}-0\right)=S^{\prime \prime \prime}\left(t_{N-1}+0\right) .
\end{aligned}
$$

It is straightforward to check that here the Schoenberg-Whitney conditions [14, p.171] are satisfi ed, thus, this problem has the unique solution. Now, $S \in S_{3}\left(\Delta_{N}\right)$ being the solution of (3.3), we get $P_{N} f=S^{\prime}$ as a solution of (3.2) and, vice versa, for $u=P_{N} f \in S_{2}\left(\Delta_{N}\right)$ being a solution of (3.2), we have $S(t)=\int_{0}^{t} u(s) d s$ as a solution of (3.3). This means that projections $P_{N}$ are correctly defi ned by (3.2).

Lemma 2. Suppose the kernel $\mathcal{K}$ in (2.5) is such that the operator $K$ maps the space $C[0, T]$ into itself. Then the subdomain problem (2.2), (3.1) is equivalent to the equation

$$
u=P_{N} K u+P_{N} f, u \in S_{2}\left(\Delta_{N}\right) .
$$

The proof is analogous to that of Lemma 1.

We would like to apply general convergence theorem for operator equations. One of the assumptions in this theorem is the convergence of the sequence of approximating operators $P_{N}$ to the identity or injection operator. This means that the uniform boundedness of the sequence $P_{N}$ is the key problem in the study of collocation method (2.2), (3.1).

For given any function $f \in C[0, T]$, let us consider $u=P_{N} f$ determined by the conditions

$$
\begin{aligned}
& u(0)=f(0), \\
& \int_{t_{i-1}}^{t_{i}} u(t) d t=\int_{t_{i-1}}^{t_{i}} f(t) d t, i=1, \ldots, N, \\
& u^{\prime \prime}\left(t_{N-1}-0\right)=u^{\prime \prime}\left(t_{N-1}+0\right) .
\end{aligned}
$$

Using the notation $u_{i}=u\left(t_{i}\right)$ and $t=t_{i-1}+\tau h_{i}, \tau \in[0,1]$, we have the representation of $u$ for $t \in\left[t_{i-1}, t_{i}\right]$

$$
u(t)=6 z_{i} \tau(1-\tau)+u_{i-1}\left(1-4 \tau+3 \tau^{2}\right)+u_{i} \tau(3 \tau-2) .
$$

The continuity of the derivative $u^{\prime}$ in the knots, i.e. $u^{\prime}\left(t_{i}-0\right)=u^{\prime}\left(t_{i}+0\right)$, gives

$$
\mu_{i} u_{i-1}+2 u_{i}+\lambda_{i} u_{i+1}=w_{i}, \quad i=1, \ldots, N-1,
$$


where

$$
\mu_{i}=1-\lambda_{i}=\frac{h_{i+1}}{h_{i}+h_{i+1}}, \quad w_{i}=3\left(\mu_{i} z_{i}+\lambda_{i} z_{i+1}\right) .
$$

The initial condition $u(0)=f(0)$ adds the equation $u_{0}=f(0)$, and the not-a-knot requirement at $t_{N-1}$ could be written in the form

$$
h_{N}^{2} u_{N-2}+\left(h_{N}^{2}-h_{N-1}^{2}\right) u_{N-1}-h_{N-1}^{2} u_{N}=2\left(h_{N}^{2} z_{N-1}-h_{N-1}^{2} z_{N}\right) .
$$

Then, eliminating $u_{N}$ in (3.5) with the help of (3.6), we write the equalities to determine $u_{i}, i=0, \ldots, N-1$, as follows:

$$
\left\{\begin{array}{l}
u_{0}=f(0), \\
\mu_{i} u_{i-1}+2 u_{i}+\lambda_{i} u_{i+1}=w_{i}, \quad i=1, \ldots, N-2, \\
\frac{h_{N}}{h_{N-1}} u_{N-2}+\left(1+\frac{h_{N}}{h_{N-1}}\right) u_{N-1}=\left(3+\frac{2 h_{N}}{h_{N-1}}\right) \mu_{N-1} z_{N-1}+\lambda_{N-1} z_{N} .
\end{array}\right.
$$

It is clear now that, in the system (3.7), the difference of domination in rows is 1 . Hence, if $h_{N} / h_{N-1} \leq$ const,

$$
\max _{0 \leq i \leq N-1}\left|u_{i}\right| \leq \mathrm{const} \max _{0 \leq i \leq N}\left|z_{i}\right| \leq \mathrm{const}\|f\|_{C}
$$

and, in addition,

$$
\left|u_{N}\right| \leq \mathrm{const} \max _{0 \leq i \leq N}\left|z_{i}\right| \leq \mathrm{const}\|f\|_{C} .
$$

The representation (3.4), assumption $h_{N} / h_{N-1} \leq$ const and obtained estimates allow to get

$$
\left\|P_{N} f\right\|_{C[0, T]}=\max _{1 \leq i \leq N} \max _{t \in\left[t_{i-1}, t_{i}\right]}|u(t)| \leq \mathrm{const}\|f\|_{C[0, T]}
$$

for any $f \in C[0, T]$ with a constant independent of $N$. We have proved the following

Proposition 2. Assuming that $h_{N} / h_{N-1} \leq$ const, the projections $P_{N}$ determined by (3.2) are uniformly bounded in the space $C[0, T]$.

\section{Convergence Theorem}

In this section we present a general convergence theorem for operator equations which will be applied to the equation (2.1) mainly in linear case and analyse what this theorem gives for the nonlocal method.

Let $E$ be Banach space, $\mathcal{L}(E, E)$ and $\mathcal{K}(E, E)$ spaces of linear continuous and compact operators. Suppose we have an equation

$$
u=K u+f
$$

where $K \in \mathcal{K}(E, E)$ and $f \in E$. Let it be given a sequence of approximating operators $P_{N} \in \mathcal{L}(E, E), N=1,2, \ldots$. Consider also equations 


$$
u_{N}=P_{N} K u_{N}+P_{N} f
$$

The following theorem for second kind equations may be called classical because it is one of the most important tools in the theory of approximate methods for integral equations ( $\operatorname{see}[1,2,5])$.

Theorem 1. Suppose $u=K u$ only if $u=0$ and $P_{N} u \rightarrow u$ for all $u \in E$ as $N \rightarrow \infty$. Then

1) (4.1) has the unique solution $u^{*}$;

2) there is $N_{0}$ such that, for $N \geq N_{0}$, (4.2) has the unique solution $u_{N}^{*}$;

3) $u_{N}^{*} \rightarrow u^{*}$ as $N \rightarrow \infty$;

4) there are positive constants $C_{1}$ and $C_{2}$ such that

$$
C_{1}\left\|P_{N} u^{*}-u^{*}\right\| \leq\left\|u_{N}^{*}-u^{*}\right\| \leq C_{2}\left\|P_{N} u^{*}-u^{*}\right\|
$$

Note that this theorem can be deduced from more general ones [8, 15].

Remark. Without presenting the details let us mention that, for the general equation (4.1) with a nonlinear operator $K$, it holds a counterpart of Theorem 1 ensuring the two-sided error estimate (4.3) provided the projections $P_{N}$ converge pointwise to the identity operator (see [9], Section 50.2). This theorem needs the complete continuity, i.e. continuity and compactness, of the nonlinear operator $K$ which is guaranteed for the operator (2.5) in the space $C[0, T]$ by the continuity of the kernel $\mathcal{K}(t, s, u)$ (see [16, Chapter 10, Section 1]).

Throughout this section, consider the general (not necessarily uniform) mesh $\Delta_{N}$ with $h=\max _{1 \leq i \leq N} h_{i} \rightarrow 0$ and $h_{N} / h_{N-1} \leq$ const as $N \rightarrow \infty$. We will apply Theorem 1 to the equation (2.1) which is possible due to the following lemma.

Lemma 3. The projections $P_{N}$ defined by (3.2) converge pointwise to the identity, i.e. $P_{N} f \rightarrow f$ in $C[0, T]$ for all $f \in C[0, T]$ as $N \rightarrow \infty$.

Proof. For given $f \in C^{1}[0, T]$, let $v$ be the quadratic spline satisfying

$$
\left\{\begin{array}{l}
v(0)=f(0), \\
v\left(t_{i-1}+\frac{h_{i}}{2}\right)=f\left(t_{i-1}+\frac{h_{i}}{2}\right), \quad i=1, \ldots, N, \\
v^{\prime \prime}\left(t_{N-1}-0\right)=v^{\prime \prime}\left(t_{N-1}+0\right) .
\end{array}\right.
$$

Taking into account $\left\|P_{N}\right\| \leq$ const and $\|v-f\|_{C} \rightarrow 0$ (see [7]), we get

$$
\begin{aligned}
\left\|P_{N} f-f\right\|_{C} & \leq\left\|P_{N} f-v\right\|_{C}+\|v-f\|_{C} \\
& =\left\|P_{N}(f-v)\right\|_{C}+\|v-f\|_{C} \\
& \leq \mathrm{const}\|f-v\|_{C}+\|v-f\|_{C} \rightarrow 0 .
\end{aligned}
$$

This means that $\left\|P_{N} f-f\right\| \rightarrow 0$ for all $f \in C^{1}[0, T]$. Basing on Banach-Steinhaus theorem, we get the convergence of the sequence $P_{N}$ to the identity operator everywhere in the space $C[0, T]$, since $C^{1}[0, T]$ is dense in $C[0, T]$. The proof is completed. 
Taking $E=C[0, T]$,

$$
(K u)(t)=\int_{0}^{t} \mathcal{K}(t, s) u(s) d s, u \in C[0, T]
$$

and using Lemma 3, Theorem 1 directly yields

Theorem 2. Suppose the kernel $\mathcal{K}$ is such that $K$ is compact. Then the method (2.2), (3.1) is convergent in $C[0, T]$ and the estimate (4.3) holds.

Remark. The rate of convergence of the method (2.2), (3.1) for linear equations, as stated in Theorem 2, is determined by the two-sided estimate (4.3). It is known that quadratic spline histopolation projections $P_{N}$ have the property $\left\|P_{N} u-u\right\|=\mathcal{O}\left(h^{3}\right)$ for smooth functions $u$ (actually, it suffi ces $u^{\prime} \in \operatorname{Lip} 1$ ). The rate $\mathcal{O}\left(h^{3}\right)$ is confi rmed by the numerical tests presented in the next section.

\section{Numerical Tests}

In numerical tests we chose the test equation

$$
y(t)=\lambda \int_{0}^{t} y(s) d s+f(t), \quad t \in[0,1],
$$

with the exact solution $y(t)=\left(\sin t+\cos t+e^{t}\right) / 2$. We implemented also the nonlocal method for the equation (2.1) in linear case with

$$
\mathcal{K}(t, s)=t-s, \quad f(t)=\sin t
$$

and the exact solution $y(t)=\left(2 \sin t+e^{t}-e^{-t}\right) / 4$ on the interval $[0,1]$. These equations are used, e.g., in $[3,12,13]$. Actually, we calculated the error of the method as

$$
\|u-y\|_{\infty}=\max _{1 \leq n \leq N} \max _{0 \leq k \leq 10}\left|(u-y)\left(t_{n-1}+k h / 10\right)\right| .
$$

Results of numerical experiments are presented in Tables 1-3.

Table 1. Numerical results for $y(t)=\lambda \int_{0}^{t} y(s) d s+f(t)$, step-by-step method.

\begin{tabular}{lcccc}
\hline $\mathrm{N}$ & 4 & 16 & 64 & 256 \\
\hline$\lambda=-2$ & $3.28 \cdot 10^{-3}$ & 98.02 & $1.10 \cdot 10^{27}$ & $2.79 \cdot 10^{134}$ \\
$\lambda=-1$ & $2.59 \cdot 10^{-3}$ & 76.56 & $8.54 \cdot 10^{26}$ & $2.18 \cdot 10^{134}$ \\
$\lambda=1$ & $1.46 \cdot 10^{-3}$ & 45.99 & $5.17 \cdot 10^{26}$ & $1.32 \cdot 10^{134}$ \\
$\lambda=2$ & $1.02 \cdot 10^{-3}$ & 35.36 & $4.01 \cdot 10^{26}$ & $1.03 \cdot 10^{134}$ \\
\hline
\end{tabular}

\section{Acknowledgement}

Our work was supported by the Estonian Science Foundation grant 5260. 
Table 2. Numerical results for $y(t)=\lambda \int_{0}^{t} y(s) d s+f(t)$, nonlocal method.

\begin{tabular}{lcccc}
\hline $\mathrm{N}$ & 4 & 16 & 64 & 256 \\
\hline$\lambda=-2$ & $3.27 \cdot 10^{-3}$ & $6.27 \cdot 10^{-5}$ & $1.02 \cdot 10^{-6}$ & $1.61 \cdot 10^{-8}$ \\
$\lambda=-1$ & $3.11 \cdot 10^{-3}$ & $6.18 \cdot 10^{-5}$ & $1.02 \cdot 10^{-6}$ & $1.61 \cdot 10^{-8}$ \\
$\lambda=1$ & $2.77 \cdot 10^{-3}$ & $5.99 \cdot 10^{-5}$ & $1.01 \cdot 10^{-6}$ & $1.61 \cdot 10^{-8}$ \\
$\lambda=2$ & $2.60 \cdot 10^{-3}$ & $5.91 \cdot 10^{-5}$ & $1.01 \cdot 10^{-6}$ & $1.60 \cdot 10^{-8}$ \\
\hline
\end{tabular}

Table 3. Numerical results for $y(t)=\int_{0}^{t}(t-s) y(s) d s+f(t)$, nonlocal method.

\begin{tabular}{ccccc}
\hline $\mathrm{N}$ & 4 & 16 & 64 & 256 \\
\hline & $5.70 \cdot 10^{-3}$ & $9.02 \cdot 10^{-5}$ & $1.41 \cdot 10^{-6}$ & $2.20 \cdot 10^{-8}$ \\
\hline
\end{tabular}

\section{References}

[1] K. E. Atkinson. The numerical solution of integral equations of the second kind. Cambridge: Cambridge University Press, 1997.

[2] H. Brunner. Collocation methods for Volterra integral and related functional differential equations. Cambridge: Cambridge University Press, 2004.

[3] H. Brunner and P. J. van der Houwen. The numerical solution of Volterra equations. Amsterdam: North-Holland, 1986.

[4] M. E. A. El Tom. On the numerical stability of spline function approximations to solutions of Volterra integral equations of the second kind. BIT, 14, $136-143,1974$.

[5] W. Hackbusch. Integral equations: Theory and numerical treatment. Basel: Birkhäuser, 1995.

[6] H. S. Hung. The numerical solution of differential and integral equations by spline functions. MRC Tech. Report 1053, University of Wisconsin, Madison, 1970.

[7] W. J. Kammerer, G. W. Reddien and R. S. Varga. Quadratic interpolatory splines. Numer. Math., 22, 241 - 259, 1974.

[8] M. A. Krasnoselskii, G. M. Vainikko, P. P. Zabreiko, Y. B. Rutitskii and V. Ya. Stecenko. Approximate solution of operator equations. Groningen: Wolters-Noordhoff, 1972.

[9] M. A. Krasnoselskii and P. P. Zabreiko. Geometric methods of nonlinear analysis. Moscow: Nauka, 1975. (In Russian)

[10] P. Oja. Stability of collocation by smooth splines for Volterra integral equations. In: T. Lyche and L. L. Schumaker(Eds.), Mathematical methods for curves and surfaces, Nashville: Vanderbilt University Press, Oslo 2000, 405 - 412, 2001.

[11] P. Oja. Stability of the spline collocation method for Volterra integral equations. $J$. Integral Equations Appl., 13, 141 - 155, 2001.

[12] P. Oja and D. Saveljeva. Cubic spline collocation for Volterra integral equations. Computing, 69, 319 - 337, 2002.

[13] P. Oja and D. Saveljeva. Quadratic spline collocation for Volterra integral equations. $Z$. Anal. Anwendungen, 23, 833 - 854, 2004.

[14] L. Schumaker. Spline functions: Basic theory. NY: Wiley, 1981.

[15] G. Vainikko. Funktionalanalysis der Diskretisierungsmethoden. Leipzig: Teubner, 1976.

[16] P. P. Zabreiko and A. I. Koshelev et al. Integral Equations. Moscow: Nauka, 1968. (In Russian) 